\title{
Low Anisakis-specific IgE prevalence in dyspeptic patients in Italy - a retrospective study.
}

\author{
Fabrizio Bruschi ${ }^{1 \star}$, Barbara Castagna $^{1}$, Francesca Mori-Ubaldini $^{1 \star *}$, Barbara Pinto $^{1}$, \\ Francesco Costa $^{3}$ and Ferruccio Bonino ${ }^{2}$
}

\begin{abstract}
The aims of this case-control study were to determine the prevalence of Anisakis-specific IgE in patients reporting chronic or acute gastrointestinal (GI) symptoms and to investigate the correlation with raw fish ingestion habits.

A group of patients undergoing gastric endoscopy and a control group of healty subjects answered a self-administered questionnaire on their food habits, presence of symptoms (both allergic and not allergic), and general life style. The presence of anti-Anisakis IgE has been evaluated using a serum immunoCAP assay.

Our data show a low prevalence of IgE directed against Anisakis allergens in Italy in dyspeptic patients, despite the high consumption of poorly cooked fish. These findings does not correlate with the results of studies from other Mediterranean countries, such as Spain, for example.

The general prevalence of Anisakis allergens sensitization in Italy could be further investigated through screenings in the allergic population, especially on those patients who claim to have developed a fish allergy and with history of raw fish consumption. Moreover, the attention should be moved on recent allergic reactions associated with fishing ingestion. This could in fact indicate a recent encounter with the parasite.

Finally, we must underline that the evaluation of Anisakis-specific IgG would have probably shown a difference in terms of exposure between the two groups; thus, it might be useful to detect also this antibody class in future population-based studies.
\end{abstract}

Keywords: Anisakis, food allergy, raw fish, sensitization

'Department of Translational Research, University of Pisa, Pisa, Italy

${ }^{2}$ Department of Clinical and Experimental Medicine, Università di Pisa, Pisa, Italy Present position: Institute for Health, University of Pittsburgh Medical Center (UPMC) Chianciano Terme, Institute of Biostructure and Bioimaging (IBB), National Research Council (CNR), Naples, Fondazione Italiana Fegato (FIF), Area Science Park, Campus Basovizza, Trieste, Italy

${ }^{3}$ University Gastroenterology Unit, AOU Pisana, Pisa, Italy

**present position: Resident in the School of Infectious Diseases, University of Sassari, Sassari, Italy

*Corresponding author: F. Bruschi E-mail: fabrizio.bruschi@med.unipi.it

DOI: 10.2478/ebtj-2018-0009

\section{Introduction}

Anisakis simplex is a parasitic nematode belonging to the Anisakidae family. The life cycle of this worm involves crustaceans, fishes and marine mammals. Humans are infected as accidental hosts by eating raw or undercooked seafood that is contaminated with the parasite, causing a zoonosis called anisakidosis or anisakiasis. Within a few hours after the ingestion of a living third stage larvae (L3), the parasite causes an acute infection often associated with GI symptoms such as abdominal pain, nausea, vomiting, and/or diarrhea. The clinical picture ranges from being asymptomatic to requiring emergency room care. These clinical reactions correspond to the worm invasion of the gastrointestinal mucosae and submucosae, causing initially a hemorrhagic lesion, then inducing the formation of a pathognomonic eosinophilic phlegmon. In addition, IgE-mediated allergic reactions are often reported, as the result of a sensitization to a range of allergenic proteins. However, some patients develop simultaneously both the gastrointestinal and the allergic syndrome after ingesting living parasites. This condition would be termed "gastroallergic anisakiasis". Allergic symptoms vary from angioedema or urticaria to anaphylactic shock. The allergic manifestations may occur also after the ingestion of dead worms present in cooked fish if the patient is already sensitized by A. simplex antigens (1). 
Sensitization is commonly diagnosed through a serological assay detecting specific anti-Anisakis IgE by the immunoCAP test. If the larva is not expelled through vomit or by surgical removal, a chronic granuloma surrounding the dead parasite is generated. Thus, disease may present with chronic GI symptoms (dyspepsia, colic pain, or complicate in acute abdomen) (2). The upper GI tract, such as stomach and duodenum, is the section more commonly affected by the infection (3). However, there have been reports of cases involving the ascending colon and the sigma (4). Infection of the sigmoid colon is considered a risk factor for the development of colorectal cancer (5).

The aims of this study were to determine the serum prevalence of anti-Anisakis IgE in patients reporting chronic or acute GI symptoms and to investigate the correlation with food habits, presence of symptoms (both allergic and not allergic), and life style. Participants to the study included patients undergoing gastric endoscopy and healthy subjects (control group). All participants answered a questionnaire about fish consumption habits and other risk factors for GI symptomatology. The presence of specific anti-Anisakis IgE has been evaluated using a serum immunoCAP assay.

\section{Materials and Methods}

Subjects: From May 2004 to January 2005 two groups of patients were enrolled in the study. The first group (G1) included 136 patients $(60.2 \%$ men and $39.8 \%$ women, mean age 42 years, age range 21 to 78 years ) suffering from dyspeptic symptoms, coming from a gastroenterological practice or undergoing a programmed gastric endoscopy. The second group (G2) included 49 healthy individuals (control group), (mean age 41 years, age range 19 to 62 years) randomly selected from the blood donors practice. Both patients and control subjects were interviewed by an anamnestic questionnaire, and anti-Anisakis allergen specific IgE were evaluated with an immunoCAP assay in the sera of both of the groups. Signed consent was obtained from each participant in the study.

Data collection: A self-administered questionnaire was fullfilled by each participant. The interview collected information about cigarette smoking habits, coffee and alcohol intake, food intolerance, poorly cooked fish consumption (raw fish or shellfish, smoked or marinate fish) and both allergic and GI symptoms. The smoking habit was categorized into current smokers and-non-smokers. Cigarette consumption was expressed as cigarettes smoked per day and divided into two categories, $<10$ and $\geq 10$. Subjects were also divided in categories according to the number of coffee cups consumed per day $(<3, \geq 3)$. Daily amount of consumed alcoholic beverages were obtained with the same method of coffee consumption. About the allergic symptoms, the presence of angioedema/ urticaria after food ingestion, atopic status, previous episodes of anaphylactic shock, eczema, allergic asthma, allergic rhinitis, contact dermatitis, acute abdomen without certain cause, and rheumatologic manifestations were investigated, as well as the presence of hypereosinophilia, prior parasitic infections, and family history of allergic reactions. As for the gastrointestinal symptoms patients were asked to indicate their manifestations choosing between nausea, vomit, dyspepsia, loss of weight, chest pain, retrosternal, epigastric or abdominal pain, anorexia, dysphagia, pyrosis, diarrhea or others.

ImmunoCAP ${ }^{\circledR}$ Specific IgE: allergen specific immunoglobulin E (IgE) to Anisakis simplex were evaluated by the ImmunoCAP ${ }^{\circledR}$ ISAC (Phadia 1000,reagent p4 , 14-447501, Thermo Scientific), made of mixed Anisakis antigens, also containing the antigen Ani s1. P4 is mixed with $150 \mu \mathrm{l}$ of patient serum, and non-specific IgE are removed by a washing solution (code $\mathrm{n}^{\circ}$ 10-9518-01). Anti-IgE antibodies (monoclonal from mice) combined with a hydrolytic enzyme (beta-galactosidase ) are added. These bind to allergen specific IgE, if presents. After incubating for $1 \mathrm{~h}$, the sample undergoes a washing cycle, than it is incubated for further 30 ' with an enhancing solution (4-metilumbelliteril-beta-D-Galattoside). The result of the reaction is measured by fluorescence. A higher fluorescence value corresponds to a higher serum level of specific IgE. The instrument converts the fluorescence units in a concentration value based on a calibration curve. (Calibrator strip 0-100 $0 ; 0.35$ - 0.7; 3.5 - 17.5; 100 100KU/L 6 x 0,2 mi). Data are automatically double-read, and the average is calculated as the given result. A specific IgE concentration $>0.35 \mathrm{KUA} / \mathrm{L}$ was considered as a positive results.

Statistical analyses: The possible association of any oflifestyle factors (smoking habits, coffee and alcohol consumption) with seroconversion was analyzed using univariate analysis (Fisher exact test). Effects were considered to be statistically significant for $p<0.05$. We also calculated in each group the odds ratio (OR) of dyspeptic symptoms and risk factors as a measure of association between dyspepsia and consumption of uncooked or poorly cooked fish. $p$ values are showed in the tables only when significant.

\section{Results}

No difference between the two groups as regards smoke, alcohol and coffee consumption was observed (Table 1). On the contrary, the number of patients affected by atopic status and history of allergic symptomatology (asthma or rhinitis) was significantly higher in patients, compared to healthy controls $(p<0.001$ and $0.028-0.0128$, respectively)

Regarding the eating habits, we found a significant difference related to consumption of total poorly cooked fish $(\mathrm{OR}=3.48$, 1.61-7.52) whereas the consumption of raw fish is nearly the same in the two groups (Table 3 ). In particular, smoked fish and dry fish consumption showed the higher association with the first group $(\mathrm{OR}=5.32,95 \% \mathrm{CI} 2.63-10.76$; $\mathrm{OR}=5.60,95 \% \mathrm{CI} 2.70$ 11.59 , respectively). Also, the number of patients usually eating all the four different types of raw fish is higher in the first group (10\%) compared to controls (2\%). Even the number of patients consuming at least one type of raw fish is higher compared to healthy subjects $(86.8 \%$ and $65.3 \%$, respectively). 
Among the 136 patients, 71 presented with a GI symptomatology at the moment the questionnaire was administered and the serum sample taken, see Table 4 . The most common were pyrosis $(74.6 \%)$ and dyspepsia $(57.7 \%)$, followed by retrosternal and epigastric pain (38\% and 35.2\%). In 25 patients $(35.2 \%)$ pyrosis and dyspepsia coexisted.
68 patients reported additional GI symptoms, such as regurgitation and other digestive alterations (Table 5).

Only five individuals resulted positive for the presence of anti-Anisakis IgE. Three of them belonged to the first group (patient A, B and C) whereas 2 of them belonged to the control group.

Table 1. Lifestyle habits concerning smoke, alcohol and coffee

\begin{tabular}{|l|c|c|}
\hline & Patients $n(\%)$ & Healthy subjects $n$ (\%) \\
\hline $\begin{array}{l}\text { Smokers } \\
<10 \text { cigarettes/die }\end{array}$ & $34(25.0)$ & $10(20.4)$ \\
$>10$ cigarettes/die & $11(8.0)$ & $3(6.1)$ \\
\hline Alcohol consumers & $23(16.9)$ & $7(14.3)$ \\
\hline Coffee consumers & $39(28.7)$ & $12(24.5)$ \\
\hline 3 coffee/die & $85(62.5)$. & $34(69.4)$ \\
$>3$ coffee/die & $27(19.8)$ & $6(12.3)$ \\
\hline
\end{tabular}

Table 2. History of food intolerance and other allergic reactions

\begin{tabular}{|l|c|c|}
\hline & Patients $\mathbf{n}(\%)$ & Healthy subjects n (\%) \\
\hline Food intolerance & $10(7.3)$ & $6(12.2)$ \\
\hline History of food-related allergic reactions: & $21(15.4)$ & $4(8.2)$ \\
\hline $\begin{array}{l}\text { - Angioedema } \\
\text { - Urticaria }\end{array}$ & $6(4.4)$ & $2(4.1)$ \\
\hline - Both A/U & $15(11 ., 0)$ & $2(4.1)$ \\
\hline Atopic status & $5(3.7)$ & $0(0)$ \\
\hline Anaphylactic shock & $43(31.6)$ & $4(8.2)$ \\
\hline Eczema & $5(3.7)$ & $3(6.1)$ \\
\hline Allergic asthma & $27(19.7)$ & $4(8.2)$ \\
\hline Allergic rhinitis & $19(14.0)$ & $1(2.0)$ \\
\hline Contact dermatitis & $42(30.9)$ & $6(12.2)$ \\
\hline Family history of allergic symptoms & $30(22.1)$ & $7(14.4)$ \\
\hline
\end{tabular}

Table 3. Consumption of poorly or no cooked fish

\begin{tabular}{|c|c|c|c|c|}
\hline & Patients (\%) & $\begin{array}{l}\text { Healthy subjects } \\
\text { (\%) }\end{array}$ & $\boldsymbol{P}$ & OR $(95 \% \mathrm{Cl})$ \\
\hline $\begin{array}{l}\text { Total poorly cooked fish } \\
\text { consumers }\end{array}$ & $118(86.8)$ & $32(6.3)$ & 0.0023 & 3.48 (1.61-7.52) \\
\hline Raw fish & $33(24.2)$ & $14(28.6)$ & & $0.80(0.38-1.67)$ \\
\hline Smoked fish & $98(72.1)$ & $16(32.0)$ & $<0.0001$ & $5.32(2.63-10.76)$ \\
\hline Dry salted/pickled fish & $91(67.1)$ & $13(26.5)$ & $<0.0001$ & $5.60(2.70-11.59)$ \\
\hline Raw shellfish & $33(24)$ & $6(12.2)$ & & $2.29(0.89-5.88)$ \\
\hline 2 among the above & $21(15.4)$ & $9(18.4)$ & & - \\
\hline 3 among the above & $14(12.3)$ & $4(8.2)$ & & - \\
\hline 4 among the above & $17(10.0)$ & $1(2.0)$ & 0.0457 & $6.85(0.88-52.97)$ \\
\hline
\end{tabular}


The level of immuncoCAP positivity of the patients from group one is respectively of $13.8 \mathrm{KUA} / \mathrm{L}, 0.98 \mathrm{KUA} / \mathrm{L}$ and $0.88 \mathrm{KUA} / \mathrm{L}$. Patient A, the one with the highest IgE level, has an history of raw fish consumption involving all four type of poorly cooked fish, while patient B and C claims to consume only dry salted fish.

Presence of symptoms in positive patients vary from none to only GI to mainly allergic ones. Positive patients from the control group, instead, show an iCAP value of $0.51 \mathrm{KUA} / \mathrm{L}$ and $1.57 \mathrm{KUA} / \mathrm{L}$ and reported to eat respectively raw and smoked fish (C1) and raw fish (C2). They report to not suffer from any GI symptoms or allergic ones (Table 6).

\section{Discussion}

Positivity of IgE to Anisakis allergens showed no significant difference between the two groups.

These results totally differ from those obtained in a similar study carried out on dyspeptic patients in Spain (6) where the prevalence of seropositivity to Ani s 1 in those patients was found to be higher $(13.8 \%)$, compared to results $(0.42 \%$ of positivity) obtained in the population in Spain where a study was carried out with a random sample of 2801 healthy blood donors distributed in 53 geographic areas, proportional to the density of donors (7). This discrepancy cannot be ascribed to the sensitivity of our method which, conversely, lacks rather in specificity, compared to the method used by Spanish Authors (6).

Furthermore, in the paper cited above Anisakis-specific IgE resulted associated with the consumption of fish in vinegar, as well as raw and smoked fish (6). Our data suggest a low seropositivity to Anisakis allergens in Italy both in dyspeptic patients and in the general population, given that the $86.8 \%$ of patients and the $65.3 \%$ in healthy controls claims to usually consume poorly or not cooked fish. Moreover, the level of

Table 4. Distribution of symptoms and other modifications in the two Groups

\begin{tabular}{|l|c|c|c|}
\hline & Patients (\%) & Healthy subjects (\%) & 0.0011 \\
\hline Acute Abdomen & $28(20.6)$ & $1(2.0)$ & 0.0049 \\
\hline Rheumatologic manifestations & $37(2.2)$ & $4(8.2)$ & 0 \\
\hline Eosinophilia & $5(3.7)$ & $1(2.0)$ & $<0.0001$ \\
\hline Gl symptoms & $71(52.2)$ & & 0.0 \\
\hline
\end{tabular}

Table 5. Other common gastrointestinal symptoms reported by Group 1 patients

Others GI symptoms

- Regurgitation

$\mathrm{N}^{\circ}$ of patients (Group 1) $68 \mathrm{pz}(95,7 \%)$

- Digestive alterations

- Irritable Bowel Syndrome

- Others
$26(36.6 \%)$

17 (23.9\%)

$19(26.8 \%)$

$6(8.4 \%)$

Table 6. Positive patients to Anisakis sensitization

\begin{tabular}{|c|c|c|c|c|c|c|}
\hline Patient $\left(n^{\circ}\right)$ & $\begin{array}{l}\text { Raw fish } \\
\text { consumption }\end{array}$ & GI symptoms & $\begin{array}{l}\text { Allergic } \\
\text { symptoms }\end{array}$ & Others & $\begin{array}{c}\text { Food } \\
\text { intolerance }\end{array}$ & $\begin{array}{l}\text { Specific lgE } \\
\text { KUA/L }\end{array}$ \\
\hline Group $1 \mathrm{~A}$ & All 4 types & $\begin{array}{c}\text { Pyrosis, } \\
\text { Retrosternal } \\
\text { pain, digestive } \\
\text { alterations }\end{array}$ & None & $\begin{array}{l}\text { Rheumatologic } \\
\text { manifestations }\end{array}$ & No & 13.8 \\
\hline Group 1 B & Dry salted fish & None & None & Polyp removal & No & 0.98 \\
\hline Group 1 C & Dry salted fish & Pyrosis & $\begin{array}{c}\text { Angioedema, } \\
\text { Urticaria, } \\
\text { Eczema }\end{array}$ & $\begin{array}{l}\text { Eosinophilia, } \\
\text { family history } \\
\text { of allergic } \\
\text { reactions }\end{array}$ & No & 0.88 \\
\hline Group 2 C1 & $\begin{array}{c}\text { Raw and } \\
\text { smoked fish }\end{array}$ & None & None & None & No & 0.51 \\
\hline Group 2 C2 & Smoked fish & None & None & None & No & 1.57 \\
\hline
\end{tabular}


specific IgE in four out of five positive patients is actually low, and for each of them the presence of anti Ani s 1 antibodies should be confirmed by Western Blot analysis, in order to rule out false positive results which may be obtained with a highly sensitive but not very specific method such as Immunocap, as shown recently (8).

\section{Conclusions}

Specific IgE production is not strictly related to infection, but it characterises the proneness to develope a hypersensitivity state. Evaluation of Anisakis-specific IgG might probably show significant differences between the two groups, in terms of exposition to Anisakis antigens.

The general seropositivity to Anisakis in Italy could be further investigated through population-wide screenings on raw fish consumption.

Finally, we think that particular attention should be given to patients who report recent allergic reactions. This could indicate a recent encounter with the parasite and, consequently, a higher level of specific anti-Anisakis IgE in the serum.

\section{References}

1. Mattiucci S, D'Amelio S. Anisakiasis. In: Bruschi F. (Ed.) Heminth infections and their impact on global public health. Springer, Wien, 2014. pp. 325-365

2. Audicana MT, Ansotegui IJ, de Corres LF, Kennedy MW. Anisakis simplex: dangerous-dead and alive? Trends Parasitol 2002; 18 (1): 20-25.

3. del Pozo MD, Moneo I, de Corres LF, Audicana MT, Muñoz D, Fernandez E, Navarro JA, García M. Laboratory determinations in Anisakis simplex allergy. J Allergy Clin Immunol 1996; 97 (4): 977-984.

4. Audicana MT, Kennedy MW. Anisakis simplex: from obscure infectious worm to inducer of immune hypersensitivity. Clin Microbiol Rev 2008; 21 (2): 360-379.

5. Yoo MD, Kim MD, Lee JM,Kim MA, Han JK, Choi BI. The Association of Anisakiasis in the Ascending Colon with Sigmoid Colon Cancer: CT Colonography Findings. Korean J Radiol 2008; 9 (Suppl):56-60.

6. Toro C, Caballero ML. High Prevalence of Seropositivity to a Major Allergen of Anisakis simplex, Ani s1, in Dyspeptic Patients. Clin Diagn Lab Immunol 2004;11(1):115-118.

7. Valiñas B, Lorenzo S, Eiras A, Figueiras A, Sanmartín ML, Ubeira FM. Prevalence of and risk factors for IgE sensitization to Anisakis simplex in a Spanish population. Allergy 2001; 56 (7): 667-671.

8. Mattiucci S, Colantoni A, Crisafi B, Mori-Ubaldini F, Caponi L, Fazii P, Nascetti G, Bruschi F. IgE sensitization to Anisakis pegreffii in Italy. Comparison of two methods for the diagnosis of allergic anisakiasis. Parasite Immunol 2017; 39 (7):e12440. 